\title{
Lattice study of meson properties at finite temperature using the truncated overlap fermions
}

\author{
Yuko Murakami \\ Research and Development Laboratory, Seikow Chemical Engineering \& Machinery, LTD, \\ Akashi 674-0093, Japan
}

\begin{abstract}
Atsushi Nakamura
School of Biomedicine, Far Eastern Federal University, 690950 Vladivostok, Russia Theoretical Research Division, Nishina Center, RIKEN, Wako 351-0198, Japan

Research Center for Nuclear Physics (RCNP), Osaka University, Ibaraki, Osaka 567-0047, Japan
\end{abstract}

\section{Motoo Sekiguchi}

School of Science and Engineering, Kokushikan University, Tokyo 154-8515, Japan

Hiroaki Wada*

School of Science and Engineering, Kokushikan University, Tokyo 154-8515, Japan

E-mail: hrwada@kokushikan.ac.jp

\section{Masayuki Wakayama}

Center for Extreme Nuclear Matters (CENuM), Korea University, Seoul 02841, Republic of Korea

Department of Physics, Pukyong National University (PKNU), Busan 48513, Republic of Korea Research Center for Nuclear Physics (RCNP), Osaka University, Ibaraki, Osaka 567-0047,

Japan

\begin{abstract}
We studied the meson properties at finite temperature using quenched lattice QCD simulations with two-flavor truncated overlap fermions. We calculated the screening masses of four types of meson, pseudo-scalar, vector, scalar and axial-vector mesons. We observed a tendency for the screening masses in all the channels to be degenerate at high temperature, which is in accord with the effective restoration of the $U_{A}(1)$ symmetry.
\end{abstract}

37th International Symposium on Lattice Field Theory - Lattice2019

16-22 June 2019

Wuhan, China

\footnotetext{
* Speaker.
} 


\section{Introduction}

The vacuum properties of QCD are related to chiral symmetry breaking and the $U(1)_{A}$ anomaly. Chiral symmetry breaking causes the appearance of the nearly massless pseudo-scalar meson $\pi$, as a pseudo Nambu-Goldstone boson. The $U(1)_{A}$ anomaly affects the mass of $\eta^{\prime}(958)$, which is smaller than the masses of the other eight low-lying pseudo-scalar mesons, and the small mixing angle between $\eta^{\prime}(958)$ and $\eta(550)$.

Restoring chiral symmetry should lead to degeneracy of the hadron spectral functions of the chiral partners. The chiral partner of the $\pi$ is the $\sigma$ meson (isoscalar-scalar) and that of the $\rho$ meson is the $a_{1}$ (isovector-axial-vector) meson in the chiral $S U(2)_{L} \times S U(2)_{R}$ symmetry. The rate of degeneracy of the spectral functions of the chiral partners can be considered a measure of the symmetry restoration. As a feature of the QCD vacuum, a phase transition occurs around the pseudo critical temperature $T_{c}$ at zero density. The chiral symmetry is spontaneously broken at temperatures lower than $T_{c}$. Conversely, at temperatures higher than $T_{c}$, the chiral symmetry is restored. Moreover, the $U(1)_{A}$ anomaly may also be effectively restored at temperatures higher than $T_{c}$ [1].

Calculations based on chiral effective theories show that the chiral partners $\pi$ and $\sigma$ become soft and tend to become degenerate near $T_{c}$ [2, 3, 4, 5]. The QCD sum-rule shows that the masses of the $\rho$ meson and its chiral partner, the $a_{1}$ meson, both decrease at finite temperatures, although the temperature of the possible degeneracy depends on the calculation method [6]. Lattice QCD simulations have been performed [7, 9, calculating the screening masses at finite temperatures around the pseudo critical temperature and investigating the strength of the $U(1)_{A}$ anomaly. In particular, Maezawa et al. performed a dynamical QCD simulation with light up/down quarks described using the highly improved staggered fermion action with the strange quark [9]. Their results showed that the screening masses of positive parity mesons decrease whereas those with negative parity monotonically increase with temperature and thereby tend to become degenerate. The screening masses of the mesons approach $2 \pi T$ above the pseudo critical temperature whereas the $U(1)_{A}$ symmetry is broken even at $T \sim 1.5 T_{c}$.

We calculate the screening masses of chiral partners such as $\pi$ (the $\rho$ meson) and the scalar meson (the $a_{1}$ meson) at finite temperatures with the quenched truncated overlap fermion action and compare our results with the results of previous research. The paper is organized as follows. In Section 2, we briefly explain the formulation of the truncated overlap fermions. In Section 3, we describe the simulation procedure and show the results for the screening masses of the $\pi, \rho, a_{1}$ and scalar mesons. Section 4 presents some concluding remarks.

\section{Formulation}

We adopt a quark action using the truncated overlap formalism by Boriçi [10] based on the domain wall fermion action [11,12]. The truncated overlap fermion action is defined by

$$
S_{T O F}=\bar{\psi}(x) D_{T O F}(x, y) \psi(y),
$$

where $\psi$ is the fermion field and the indexes $x, y$ represent the four-dimensional lattice sites and color. The Dirac spinor subscripts are omitted. The truncated overlap fermion matrix operator 
$D_{T O F}$ is represented as

$$
D_{T O F}=\varepsilon^{\dagger} P^{\dagger} D_{P V}^{-1} D_{D W F} P \varepsilon
$$

The domain wall fermion matrix operator $D_{D W F}$ has indexes for the four-dimensional coordinates, color and Dirac spinor and also an index for the fifth-dimensional coordinate, $x_{5}$, which is in the range $1 \leq x_{5} \leq N_{5} . D_{D W F}$ is represented as an $N_{5} \times N_{5}$ matrix,

$$
D_{D W F}=\left(\begin{array}{cccccc}
D_{W F}+1 & -P_{L} & 0 & \cdots & 0 & m_{f} P_{R} \\
-P_{R} & D_{W F}+1 & -P_{L} & \ddots & \vdots & 0 \\
0 & -P_{R} & D_{W F}+1 & \ddots & 0 & \vdots \\
\vdots & 0 & \ddots & \ddots & -P_{L} & 0 \\
0 & \vdots & \ddots & -P_{R} & D_{W F}+1 & -P_{L} \\
m_{f} P_{L} & 0 & \cdots & 0 & -P_{R} & D_{W F}+1
\end{array}\right)
$$

where $D_{W F}$ is the Wilson fermion matrix operator,

$$
D_{W F}(x, y)=\left(4-M_{5}\right) \delta_{x, y}-\frac{1}{2} \sum_{\mu=1}^{4}\left[\left(1-\gamma_{\mu}\right) U_{\mu}(x) \delta_{y, x+\hat{\mu}}+\left(1+\gamma_{\mu}\right) U_{\mu}^{\dagger}(y) \delta_{y, x-\hat{\mu}}\right]
$$

and $M_{5}$ is the domain wall height. $P_{R / L}=\left(1 \pm \gamma_{5}\right) / 2$ are the projection operators and $m_{f}$ corresponds to a bare quark mass. The Pauli-Villars matrix $D_{P V}$ is defined by setting $D_{D W F}$ of Eq. 2.3. with $m_{f}=1$. The matrices $P$ and $\varepsilon$ are defined as

$$
P=\left(\begin{array}{ccccc}
P_{L} & P_{R} & 0 & \cdots & 0 \\
0 & P_{L} & P_{R} & \ddots & \vdots \\
\vdots & 0 & \ddots & \ddots & 0 \\
0 & \vdots & \ddots & P_{L} & P_{R} \\
P_{R} & 0 & \cdots & 0 & P_{L}
\end{array}\right) \quad, \quad \varepsilon=\left(\begin{array}{c}
1 \\
0 \\
\vdots \\
0 \\
0
\end{array}\right)
$$

Note that because $\varepsilon$ acts as a vector for the fifth dimension, the fermion field $\psi(x)$ does not have the fifth dimensional index.

Equation (2.2) is transformed into the four-dimensional formulation as

$$
D_{T O F}=\frac{1+m_{f}}{2}+\frac{1-m_{f}}{2} \gamma_{5} \frac{\left(1+H_{W}\right)^{N_{5}}-\left(1-H_{W}\right)^{N_{5}}}{\left(1+H_{W}\right)^{N_{5}}+\left(1-H_{W}\right)^{N_{5}}},
$$

where $H_{W}$ is defined by

$$
H_{W}=\gamma_{5} \frac{D_{W F}}{D_{W F}+2} .
$$

Because in the limits $N_{5} \rightarrow \infty$ and $m_{f} \rightarrow 0, D_{\text {TOF }}$ satisfies the Ginsparg-Wilson relation,

$$
\gamma_{5} D_{T O F}+D_{T O F} \gamma_{5}=a D_{T O F} \gamma_{5} D_{T O F}
$$

the truncated overlap fermion action has lattice chiral symmetry at the limits. Since the chiral symmetry affects the meson properties at finite temperatures, lattice chiral symmetry should be essential in the investigation of the meson properties. 


\section{Lattice Simulation}

We generate gauge configurations with the Iwasaki gauge action in quenched lattice QCD using the pseudo heat-bath algorithm. We also calculate the propagators of $q \bar{q}$ mesons which are composed of two-flavor $(u$ and $d$ ) quarks and anti-quarks with the truncated overlap fermions. The numerical simulations are performed in two-step procedures as follows. First, we search a line of constant physics in the parameter space $\left(\beta, m_{f}\right)$ for the truncated overlap fermions by simulating at zero temperature. We then obtain eight sets of $\left(\beta, m_{f}\right)$. Secondly, we calculate the screening masses of the $\pi, \rho, a_{1}$ and scalar mesons on the sets of $\left(\beta, m_{f}\right)$ at finite temperatures.

\section{Step1: Simulation at zero temperature}

To determine the bare quark masses $m_{f}$ of the truncated overlap fermions corresponding to each $\beta$, we calculate propagators of the $\pi$ and $\rho$ mesons and obtain those masses at zero temperature. We take a lattice size of $N_{s}^{3} \times N_{t} \times N_{5}=16^{3} \times 16 \times 32$ where $N_{s}, N_{t}$ and $N_{5}$ are the length of the space, temporal and fifth-dimensional axes, respectively. As shown in Table 1, we set the values of $\beta$ to the eight values used in Refs. [13, 14]. The values of the lattice space $a$ refer to the results of the quenched simulations with the Iwasaki gauge action in Ref. [14]. We generate $12-80$ quenched gauge configurations with the Iwasaki gauge action for each $\beta$. We calculate the $\pi$ and $\rho$ meson propagation functions with the truncated fermion action where we set the height of the domain wall $M_{5}$ to 1.65 and take several appropriate values for the bare quark masses $m_{f}$. The finite $N_{5}$ effect for $M_{5}=1.65$ at zero temperature was discussed in Refs. [15]. We then derive the effective masses. Figure 1 shows the dependence of the ratio of the pole masses of the $\pi$ and $\rho$ mesons, $m_{\pi} / m_{\rho}$, on the bare quark masses $m_{f}$. For the second-stage calculation at finite temperatures, the values of the bare quark masses $m_{f}$ for each $\beta$ are determined as input parameters under the condition $m_{\pi} / m_{\rho}=0.80$ in Table 1

Table 1: Simulation parameters. $m_{f}$ is defined for each $\beta$ in the Step1. The sets $\left(\beta, m_{f}\right)$ are used as input parameters in Step2. The values of $T / T_{c}$ and $a$ are reported in Refs. [13, 14]. The number of configurations in Step2 is listed in the last column.

\begin{tabular}{ccccc}
\hline$\beta$ & $T / T_{c}$ & $a \mathrm{fm}$ & $m_{f}$ & confs. \\
\hline 2.187 & 0.86 & $0.2079(15)$ & 0.177 & 2,400 \\
2.214 & 0.91 & $0.1977(13)$ & 0.175 & 2,000 \\
2.247 & 0.97 & $0.1853(09)$ & 0.160 & 2,000 \\
2.281 & 1.04 & $0.1727(10)$ & 0.150 & 2,000 \\
2.334 & 1.14 & $0.1577(09)$ & 0.145 & 2,000 \\
2.416 & 1.32 & $0.1359(07)$ & 0.133 & 2,000 \\
2.487 & 1.59 & $0.1206(09)$ & 0.113 & 2,000 \\
2.575 & 1.69 & $0.1065(07)$ & 0.104 & 2,000 \\
\hline
\end{tabular}




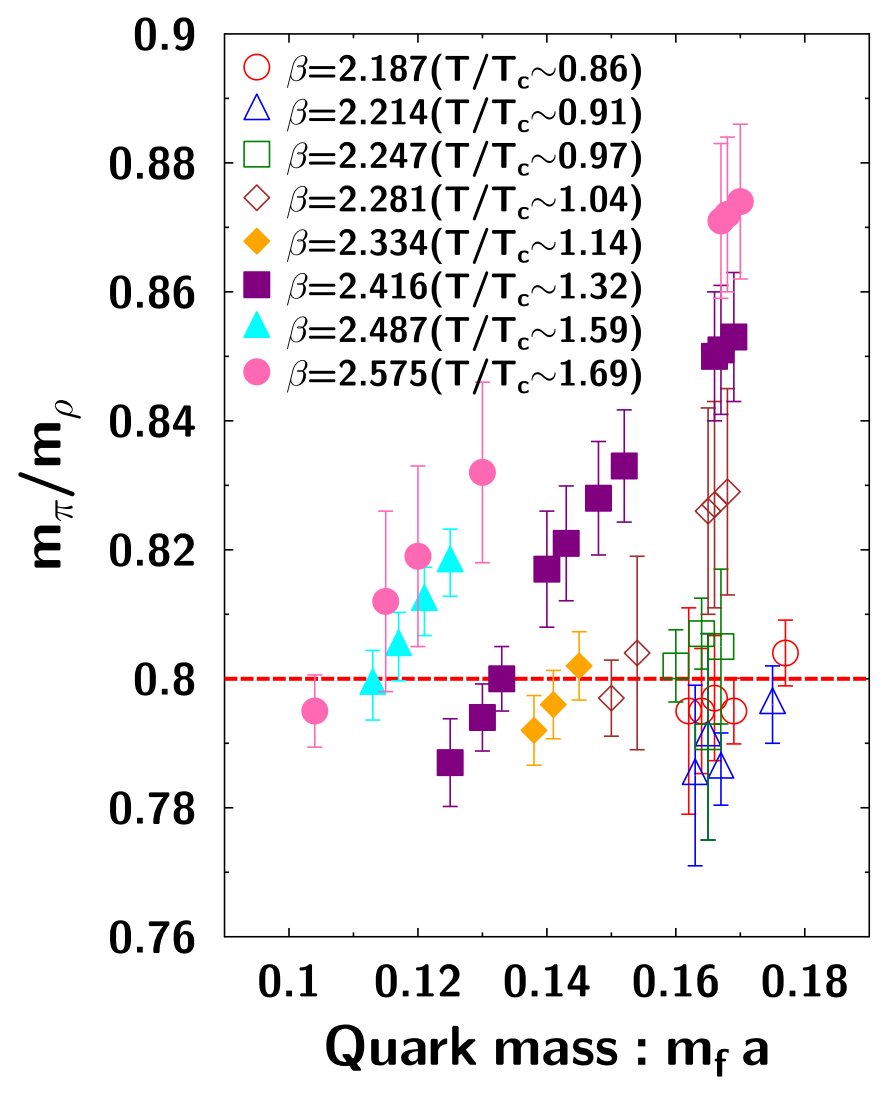

Figure 1: Ratio $m_{\pi} / m_{\rho}$ versus the bare quark mass $m_{f}$.

$\underline{\text { Step2: Simulation at finite temperature }}$

We calculate the screening masses of mesons at finite temperatures with a lattice size of $16^{3} \times 4$ and a length of the fifth dimension of $N_{5}=32$. The length of the temporal axis in Step2 is smaller than it is in Step1. The values of $\beta$ and $m_{f}$ are listed in Table 1 and the height of the domain wall $M_{5}=1.65$ is same as the simulation in Step1. We generate 2,000-2,400 gauge configurations for each $\beta$ and calculate the screening masses of the $\pi, \rho$, scalar and axial-vector mesons, considering only the connected diagram in the scalar channel. Figure 2 shows the temperature dependence of the screening mass of each meson. The vertical axis of Fig. 2 2 represents the ratios of the screening mass at finite temperature, $M_{\text {meson }}(T)$, to the pole mass of the $\rho$ meson at zero temperature, $M_{\rho}(0)$. The horizontal axis of Fig. 2 represents the ratio of $T$ to $T_{c}$, which is defined by the peak of the susceptibility of the $Z(3)$-rotated Polyakov line in Ref. [13]. In the low-temperature region, $T<T_{c}$, the screening masses of the $\pi, \rho$ and scalar mesons are unchanged. For the pseudo-vector meson $a_{1}$, the statistical error is too large to obtain the screening mass. At temperatures higher than the pseudo critical temperature, $T>T_{c}$, the screening masses of all mesons increase monotonically with $T$. We also find that the chiral partners are degenerate at high temperature: $M_{\pi} \sim M_{\text {scalar }}$ and $M_{\rho} \sim M_{a_{1}}$. 


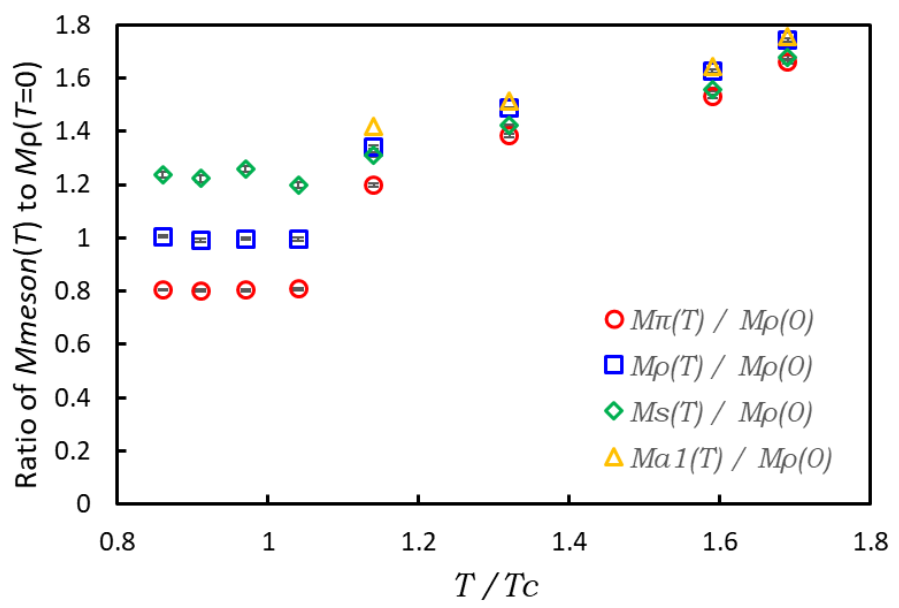

Figure 2: Screening mass ratio of $M_{\text {meson }}(T)$ to $M_{\rho}(0)$ versus $T / T_{c}$ in our results.

Figure 3 is generated based on the result of the previous work by Maezawa et al. [9]. Their simulation was performed with the highly improved staggered fermion action and the tree-level improved gauge action. The up/down quarks are physically light and treated as dynamical quarks in their simulation. The result of Fig. 3 is obtained with a light quark mass corresponding to $m_{\pi} \sim 160$ $\mathrm{MeV}$ at zero temperature. Note that they simulated the scalar meson with only the connected diagram. In Fig. 3, the curves of the screening masses of the scalar and $a_{1}$ mesons are convex downward at low temperature. However, in our result, the screening mass of the scalar meson is unchanged at low temperature. The disagreement in the results may be due to the difference in the fermion actions or the bare quark masses. On the other hand, in Fig. 3] a mass gap between $\pi$ (the scalar meson) and the $\rho$ meson (the $a_{1}$ meson) is seen at high temperature, which means that the $U(1)_{A}$ symmetry is broken until $T \sim 1.5 T_{c}$. In contrast, our results show that the $U(1)_{A}$ symmetry is mostly restored at high temperatures.

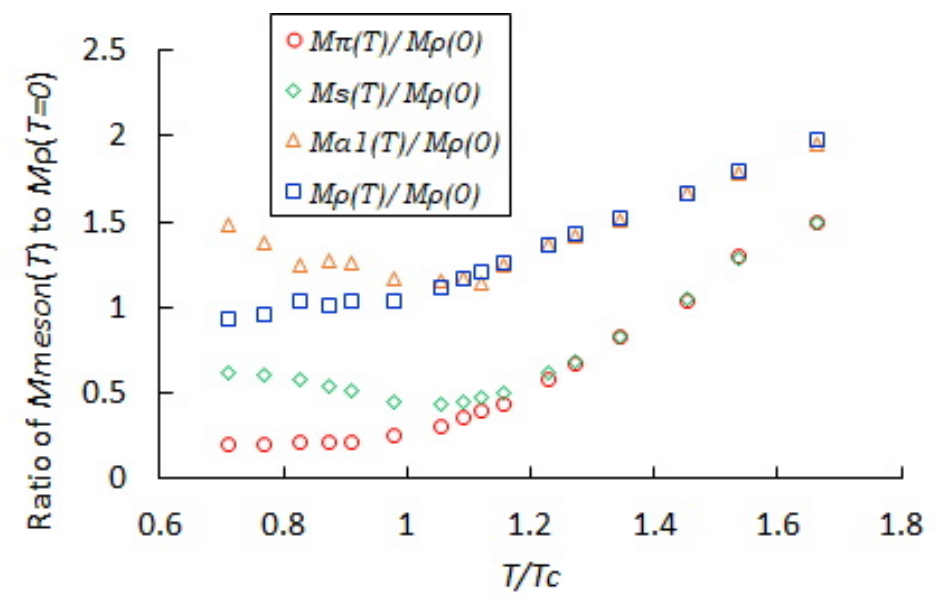

Figure 3: Screening mass ratio of $M_{\text {meson }}(T)$ to $M_{\rho}(0)$ versus $T / T_{c}$ of previous work $\square$. 


\section{Summary}

We calculated the screening masses at finite temperatures with the quenched truncated overlap fermion action. To obtain input parameters for calculations at finite temperatures, we searched a line of constant physics in the parameter space $\left(\beta, m_{f}\right)$ at zero temperature. We found that the screening masses of the $\pi, \rho$ and scalar mesons are almost independent of temperature at low temperatures below $T_{c}$. At high temperatures, the screening masses of all mesons increase with temperature. Moreover, the screening masses of $\pi$ and the scalars ( $\rho$ and $a_{1}$ ) tend to be degenerate. Our results differ from previous works in the low-temperature region. In particular, the screening mass of the scalar meson is almost constant at low temperatures in our results. The disagreement may be due to the difference of the fermion actions or the bare quark masses. Therefore, calculations of light quarks with the truncated overlap fermion action are planned as a future work.

\section{Acknowledgment}

This work was supported by RSF grant 15-12-20008 and the National Research Foundation of Korea (NRF) grant funded by the Korean government (MSIT) (No. 2018R1A5A1025563).

The simulations were performed on the supercomputer system SX-ACE at RCNP and the Cybermedia Center, Osaka University, and were conducted using the Fujitsu PRIMEHPC FX10 System (Oakleaf-FX, Oakbridge-FX) at the Information Technology Center, The University of Tokyo. This work was supported by "Joint Usage/Research Center for Interdisciplinary Largescale Information Infrastructures" in Japan (Project ID: jh180053-NAJ and jh190048-NAH).

\section{References}

[1] R. D. Pisarski and F. Wilczek, Phys. Rev., D29, 338,1984

[2] T. Hatsuda and T. Kunihiro, Phys. Lett., B145, 7,1984

[3] T. Hatsuda and T. Kunihiro, Phys. Rev. Lett., 55, 158, 1985

[4] T. Hatsuda and T. Kunihiro, Prog. Theor. Phys., 74, 765, 1985

[5] T. Hatsuda and T. Kunihiro, Phys. Lett., B185, 304 , 1987

[6] T. Hatsuda, Y. Koike and S. H. Lee, Nucl. Phys., B394, 221, 1993

[7] A. Bazavov, F. Karsch, Yu Maezawa, S. Mukherjee, and P. Petreczky, Phys. Rev., D91, 5, 2015

[8] B. B. Brandt, A. Francis, H. B. Meyer, O. Philipsen, D. Robaina, and H. Wittig, JHEP, 12, 158 , 2016

[9] Y. Maezawa, F. Karshc, S. Mukherjee and P. Petreczky, PoS, LATTICE2015:199, 2016

[10] A. Borici, Nucl. Phys. Proc. Suppl., 83, 771, 2000

[11] D. B. Kaplan, phys. Lett.. B288, 342, 1992

[12] V. Furman and Y. Shamir, Nucl. Phys., B439, 54, 1995

[13] M. Okamoto et al. (CP-PACS Collaboration), Phys. Rev. D60, 094510, 1999

[14] A.A. Khan et al. (CP-PACS Collaboration), Nucl. Phys. B ( Proc. Suppl. ), 83, 176, 2000

[15] M. Wakayama, Y. Murakami, S. Muroya, A. Nakamura, C. Nonaka, M. Sekiguchi and H. Wada, JPS Conf. Proc. 26, 031007 (2019). 